\section{Avaliação da assistência ao paciente com diabetes e/ou hipertensão pelo Programa Saúde da Família do Município de Francisco Morato, São Paulo, Brasil}

\author{
Healthcare assessment for patients with diabetes \\ and/or hypertension under the Family Health \\ Program in Francisco Morato, São Paulo, Brazil
}

Daniela Cristina Profitti de Paiva 1

Ana Aparecida Sanches Bersusa 1

Maria Mercedes L. Escuder 1

\footnotetext{
1 Núcleo de Investigação e Estudos em Epidemiologia, Instituto de Saúde, São Paulo, Brasil.

Correspondência A. A. S. Bersusa Núcleo de Investigação e Estudos em Epidemiologia, Instituto de Saúde. Rua Santo Antonio 590 São Paulo, SP

01314-000, Brasil. anabersusa@ig.com.br
}

\begin{abstract}
The Brazilian Ministry of Health launched its Family Health Program (FHP) in 1994 as a new strategy aimed at reorganizing the healthcare system starting from the primary care level. The Program prioritizes delivery of care to groups identified as presenting increased risk, such as diabetic and hypertensive individuals. The objective of this study was to evaluate the care delivered to such patients by professionals in this program in the municipality of Francisco Morato, São Paulo, Brazil. A random cluster-based sample selected 84 patients with diabetes and/or hypertension who answered a questionnaire at home. Seventy-two patients responded. Of these, $19 \%$ were diabetic, $41.7 \%$ hypertensive, and $38.9 \%$ had both diabetes and hypertension. As for the care offered by the FHP, only $35.9 \%$ received excellent diagnostic workups and $10.9 \%$ excellent physical examinations. Some $20.8 \%$ of the patients reported illness-related complications. With the implementation of the FHP, access to care improved for both diabetic and hypertensive patients. The FHP proved to be a valid alternative to increase healthcare access, seeking to achieve greater equity by delivering care in accordance with the population's needs.
\end{abstract}

Hypertension; Diabetes Mellitus; Patient Care

\section{Introdução}

Nas últimas décadas, houve uma importante mudança no perfil da mortalidade da população brasileira, com aumento dos óbitos causados por doenças crônico-degenerativas e causas externas. As doenças cardiovasculares são as causas mais comuns de morbidade e mortalidade em todo o mundo e, entre os fatores de risco para doença cardiovascular, encontramse o diabetes mellitus e a hipertensão arterial, fatores independentes e sinérgicos 1,2 .

O estudo multicêntrico sobre prevalência de diabetes mellitus no Brasil 3,4 apontou um índice de $7,6 \%$ na população brasileira entre 30-69 anos, atingindo cifras próximas a $20 \%$ na população acima dos 70 anos. Cerca de $50 \%$ dessas pessoas desconhecem o diagnóstico, e 25\% da população diabética não fazem nenhum tratamento.

Outro importante problema de saúde pública é a hipertensão arterial, cuja prevalência estimada na população brasileira adulta é de cerca de 15 a $20 \%$, sendo que, entre a população idosa, esta cifra chega a $65 \%$. Entre os hipertensos, cerca de $30 \%$ desconhecem serem portadores da doença. É uma doença que apresenta alto custo social, sendo responsável por cerca de $40 \%$ dos casos de aposentadoria precoce e de absenteísmo no trabalho 5,6.

O controle metabólico rigoroso associado a medidas preventivas e curativas relativamente 
simples são capazes de prevenir ou retardar o aparecimento das complicações crônicas do diabetes mellitus, resultando em melhor qualidade de vida ao indivíduo diabético. Da mesma forma, o controle da hipertensão arterial resulta na redução de dano aos órgãos-alvo. Para o controle de ambas as patologias, são necessárias medidas que envolvem mudanças no estilo de vida do indivíduo 6,7. O manejo do diabetes mellitus e da hipertensão arterial deve ser feito dentro de um sistema hierarquizado de saúde, sendo sua base o nível primário de atendimento 8 .

Frente à crise vivida no setor saúde, o Ministério da Saúde, em 1994, implantou o Programa Saúde da Família (PSF), com o objetivo de proceder à reorganização da prática assistencial a partir da atenção básica, em substituição ao modelo tradicional de assistência, orientado para a cura de doenças. Assim sendo, o PSF pretende promover a saúde através de ações básicas que possibilitam a incorporação de ações programáticas de forma mais abrangente 5,9 .

A dinâmica proposta pelo PSF, centrada na promoção da qualidade de vida e intervenção nos fatores que a colocam em risco, permite a identificação mais acurada e um melhor acompanhamento dos indivíduos diabéticos e hipertensos 5,9 .

Dessa forma, o presente estudo tem como objetivo descrever o perfil da população diabética e/ ou hipertensa coberta pelo PSF do Município de Francisco Morato, São Paulo, Brasil, e avaliar a assistência prestada a essa população. A opção pela abordagem conjunta do diabetes mellitus e da hipertensão arterial no presente estudo foi baseada nos seguintes aspectos comuns às duas patologias: etiopatogênia, fatores de risco, importância do tratamento não medicamentoso, cronicidade, complicações, ocorrência geralmente assintomática, difícil adesão ao tratamento, necessidade de controle rigoroso para evitar complicações e de acompanhamento por equipe multidisciplinar, além de ambas serem patologias facilmente diagnosticadas 10 .

\section{Material e método}

Este estudo faz parte de uma pesquisa avaliativa do PSF de Francisco Morato, desenvolvido pelo Instituto de Saúde da Secretaria de Estado da Saúde de São Paulo. Tomou-se o conceito de Contandriopoulos et al. 11 de pesquisa avaliativa no qual são utilizados métodos científicos para se proceder com um julgamento ex-post de uma intervenção, fornecendo instrumentos para ajudar na tomada de decisões. Avaliar é medir, comparar e ter fundamentos para emitir juízo de valor.

O Município de Francisco Morato foi escolhido como área de estudo devido à proximidade com o Município de São Paulo, ao interesse dos gestores locais, ao tempo de implantação do PSF e à alta cobertura do programa.

O estudo enfoca a avaliação do PSF a partir da opinião dos usuários. Aplicou-se um inquérito domiciliar, com a finalidade de entrevistar usuários cadastrados nos programas de diabetes mellitus e hipertensão arterial de Francisco Morato. Segundo Cesar \& Tanaka 12, é possível definir com maior especificidade diretrizes e estratégias para implantação de um sistema de saúde usando-se estudos com base populacional, na busca de maior eqüidade no atendimento às necessidades de saúde da população.

A implantação do PSF no município teve início em janeiro de 2000. Em 31 de agosto de 2001, o programa contava com 15 Equipes de Saúde da Família (ESF) atuando em dez Unidades de Saúde da Família (USF), divididas em dez setores, tendo 29.465 famílias cadastradas, correspondendo a 119.776 pessoas.

Segundo dados do Sistema de Informação em Atenção Básica (SIAB) do município, nessa data, estavam cadastrados 1.634 diabéticos, sendo $88,1 \%$ desses acompanhados pelas ESF e 7.577 hipertensos cadastrados, com acompanhamento de 87,3\%. Essas informações foram usadas para o cálculo do tamanho da amostra necessário para o desenvolvimento do estudo. Aceitou-se um erro (d) de 7,0 pontos percentuais, em um nível de confiança de $95 \%$ e adotou-se a estimativa de $88 \%$ de pacientes em acompanhamento pelas equipes de PSF. Com esses parâmetros, chegou-se a uma amostra de 83 pacientes 13. Questões de tempo e de custo para o desenvolvimento das entrevistas foram relevantes nesse cálculo. Considerou-se que, como as entrevistas seriam realizadas durante o dia, poderia haver uma importante perda por ausência do entrevistado, visto que o município é caracterizado como cidade-dormitório e, então, acrescentou-se $25 \%$ ao tamanho original, o que resultou no tamanho final da amostra de 105 pacientes. Com o cadastro no SIAB dos pacientes ordenados por tipo de patologia (hipertensão arterial e diabetes mellitus) e por equipe de saúde da família, procedeu-se o sorteio sistemático 14 , com a intenção de se obter uma amostra proporcional ao tipo de patologia e à unidade de saúde.

A proporção de pacientes usuários ativos do PSF e suas características quanto ao sexo e 
faixa etária estão apresentadas acompanhadas de seus respectivos intervalos de confiança, com a finalidade de comparar a população amostrada com os dados cadastrais.

Escolaridade, ocupação e renda familiar em salários mínimos foram usadas para descrever o perfil da população estudada em Francisco Morato, assim como a proporção dos pacientes classificados como graves, segundo registro da primeira consulta no prontuário.

A avaliação da qualidade da assistência foi vista nos seguintes aspectos: (1) uso do serviço; (2) qualidade da consulta e (3) estilo de vida.

\section{Uso do serviço}

A avaliação segundo o uso do serviço foi realizada por meio das questões:

- Tempo (em dias) decorrido entre o reconhecimento da doença pela ESF e o agendamento da primeira consulta: avaliação da agilidade do atendimento;

- Acesso ao serviço público de saúde local antes e depois da implantação do PSF: avaliação das diferenças entre o modelo tradicional de atenção básica e o PSF;

- Acesso ao medicamento prescrito: avaliação da resolutividade do serviço, envolvendo questões de como o paciente obteve o medicamento e, no caso de diabéticos, o material para aplicação da insulina. Comparou-se se o medicamento (nome genérico) prescrito pelo médico, registrado no prontuário, coincidia com o medicamento mostrado pelo paciente no momento da entrevista;

- Satisfação do paciente: apresentada em proporção de pacientes que responderam positivamente se houve resolutividade para seu problema e se ele se considera satisfeito com a assistência atual.

\section{Qualidade da consulta}

Tendo como referenciais teóricos o III Consenso Brasileiro de Hipertensão Arterial 6, o Consenso Brasileiro sobre Diabetes 3 e o Manual de Hipertensão Arterial e Diabetes Mellitus 10, foram elaboradas questões sobre a anamnese, exame físico básico e as orientações recebidas, que devem ser realizados no momento da consulta. Considerou-se a abordagem desses aspectos tanto na consulta médica como na de enfermagem.

- Avaliação da anamnese: esse item foi composto por nove questões. Indagou-se ao paciente se, durante alguma das consultas realizadas, o profissional de saúde havia lhe pedido informações sobre a história familiar, pregres- sa e atual da doença, uso de medicamentos, estilo de vida, hábito alimentar e características do trabalho. Criou-se um escore, no qual cada resposta positiva valia um ponto. Para a análise, foram criadas três categorias, referentes ao número de respostas afirmativas do paciente: anamnese insatisfatória (0 a 2 pontos), anamnese incompleta (3 a 6 pontos) e anamnese óti$m a$ (7 a 9 pontos);

- Avaliação do exame físico: o item exame físico foi composto por sete questões que englobaram, em qualquer das consultas efetuadas pela ESF, verificação da pressão arterial (mostrou-se o esfigmomanômetro ao paciente com demonstração da técnica), peso e altura, perímetro abdominal, ausculta cardíaca e pulmonar (mostrou-se o estetoscópio ao paciente, dramatizando a técnica), exame abdominal, vascular periférico e de fundo de olho. Contando um ponto para cada resposta positiva, foram criadas três categorias, referentes ao número de respostas afirmativas do paciente: exame físico insatisfatório (0 a 2 pontos), exame físico incompleto ( 3 a 5 pontos) e exame físico ótimo (6 a 7 pontos);

- Avaliação sobre orientações recebidas: medidas como proporções de orientações quanto à dieta adequada à sua condição física, prática de atividade física, medidas de redução de estresse, importância sobre parar de fumar e o consumo de bebidas alcoólicas, em qualquer das consultas médicas ou de enfermagem, palestras, aulas ou atividades de grupo organizadas pela ESF;

- Satisfação do paciente: medida como proporção dos que referiram estar satisfeitos com o programa.

\section{Estilo de vida}

Foram analisadas questões sobre os hábitos praticados pelos usuários: dieta, atividade física e hábitos de fumar e/ou beber. A dieta alimentar foi classificada posteriormente em adequada, parcialmente adequada e inadequada, segundo recomendações do Consenso Brasileiro sobre Diabetes e do Manual de Hipertensão Arterial e Diabetes Mellitus, anteriormente citados. Buscou-se conhecer a proporção de indivíduos que responderam praticar regularmente a atividade física aeróbica.

A coleta de dados ocorreu nos meses de junho e julho de 2002. Retornou-se à casa dos pacientes não encontrados na primeira visita apenas uma vez. Nessa época, o PSF de Francisco Morato estava passando por reformulações estruturais. Uma das ESF foi transformada em Programa de Agentes Comunitários de Saúde 
(PACS), outra ESF estava incompleta, mantendo apenas os agentes comunitários de saúde (ACS). Apesar dessas dificuldades, a amostra não foi alterada, e os pacientes foram entrevistados.

Para a digitação e análise dos dados coletados, foi utilizado o programa Epi Info 6 (Centers for Disease Control and Prevention, Atlanta, Estados Unidos). Trata-se de um estudo descritivo, no qual os resultados serão apresentados em números absolutos e percentuais. A precisão e a confiabilidade da amostra estudada serão analisadas apresentando intervalos de confiança em nível de 95\% das informações sobre o uso do PSF e perfil de sexo e faixa etária predominantes.

\section{Resultados e discussão}

\section{Perfil dos entrevistados}

Dos 105 pacientes sorteados, 33 não foram entrevistados. Destes, 25 não se encontravam em casa nas duas visitas realizadas, e oito se recusaram a participar da pesquisa. Das 72 pessoas entrevistadas, 88,9\% (IC95\%: 81,5-96,3) são usuárias ativas do PSF. Entre os entrevistados não usuários do serviço, quatro têm plano de saúde.

A população estudada neste artigo é composta por 14 indivíduos com diabetes mellitus, trinta com hipertensão arterial e 28 portadores das duas patologias. A maior concentração está no sexo feminino $(68,1 \%)$ (IC95\%: $57,0-79,1)$ na faixa etária de 31 a 60 anos, com $65,3 \%$ (IC95\%: 54,0-76,5). Em relação ao sexo, os dados deste estudo são compatíveis com os registros do SIAB, que mostram que $65,2 \%$ dos diabéticos e $69 \%$ dos hipertensos cadastrados em Francisco Morato são mulheres. Quanto à faixa etária, 70,2\% dos pacientes cadastrados por hipertensão arterial sistólica ou diabetes mellitus têm entre 31 e 60 anos.

Entre os 72 entrevistados, observou-se baixa escolaridade $(22,2 \%$ analfabetos e $47,2 \%$ com 1 a 4 anos de estudo completos) e baixa renda (31,0\% renda familiar entre 1 e 2 salários mínimos e 15,5\% entre 0 e 1 salário mínimo). Quanto à ocupação, $40,3 \%$ são donas-de-casa; $22,2 \%$, aposentados ou pensionistas; $12,5 \%$, domésticas. Nenhum dos indivíduos entrevistados é natural do município estudado, sendo que $53,5 \%$ são nascidos na Região Sudeste do país, e $42,3 \%$, na Região Nordeste. Vale ressaltar que se trata de um município dormitório da grande São Paulo, que "carece de arrecadação própria, com a menor renda per capita do Estado. Só recebe o IPTU, cuja inadimplência é alta" 15.
Entre os 52 pacientes com hipertensão, 25 $(43,1 \%)$ foram classificados como hipertensos moderados e graves. Em relação aos 42 pacientes com diabetes, $24(57,1 \%)$ têm registros no prontuário de níveis glicêmicos acima de $126 \mathrm{mg} / \mathrm{dl}$. Dos 64 pacientes atendidos pelo PSF, 14 (21,9\%) afirmaram já ter apresentado alguma complicação crônica da patologia, e 26 $(40,6 \%)$ referiram já ter passado por internação hospitalar em decorrência do diabetes mellitus e/ou da hipertensão arterial.

No decorrer da coleta de dados, verificouse que, no prontuário de oito pacientes, não havia registro de glicemia de jejum, apenas de glicemia capilar, que foi então a considerada. A avaliação da glicemia através do sangue capilar com o uso de fitas reagentes é um método rápido e fácil, que pode ser utilizado para o rastreamento do diabetes mellitus, porém o diagnóstico deve ser confirmado através da glicemia plasmática de jejum 10 .

As questões referentes à qualidade do atendimento oferecido pela ESF aos diabéticos e/ou hipertensos do município foram respondidas por 64 indivíduos atendidos pelas dez unidades de PSF de Francisco Morato.

\section{Acesso ao serviço de saúde e resolutividade}

Entre os 64 usuários do PSF, 17 (26,6\%) referiram que não tinham acesso a nenhum serviço de saúde antes da implantação do programa. Entre os indivíduos que tinham acesso, 25 $(53,2 \%)$ se deslocavam para unidades básicas de saúde ou hospitais de outros municípios, e apenas $15(31,9 \%)$ recebiam atendimento em programas de atenção básica local (Tabela 1).

Recomenda-se que de 60 a $80 \%$ dos casos de diabetes mellitus e hipertensão arterial sejam tratados na rede básica municipal 10, porém é necessário que o atendimento seja resolutivo e de qualidade. Diversos estudos são realizados com o intuito de subsidiar a melhoria da cobertura e da adesão dos pacientes com hipertensão e diabetes aos tratamentos. Diamant et al. 16, em um estudo de avaliação do impacto da implantação de serviço gratuito de atenção ao adulto portador de doenças crônicas em Los Angeles, Estados Unidos, concluem que, para isso, não é suficiente divulgar o nome e o endereço da equipe de saúde. É necessário um esforço agressivo para aumentar a utilização dos serviços. O PSF coloca como prioridade na elaboração do projeto de implantação que todos tenham acesso aos serviços de saúde, a fim de garantir o princípio de igualdade a todos os cidadãos, e isso se traduz na necessidade de expansão da rede básica para as- 
sistir periferias urbanas e zonas rurais em municípios 9.

Para o tratamento do diabetes mellitus e da hipertensão arterial, são imprescindíveis a vinculação do paciente às unidades de atendimento, a garantia do diagnóstico e o atendimento por profissionais atualizados, uma vez que seu diagnóstico e controle evitam complicações ou, ao menos, retardam a progressão das já existentes. Além disso, o maior contato com o serviço de saúde promove maior adesão ao tratamento 10,17 .

Em relação ao acesso a medicamentos prescritos aos 64 entrevistados, em $54,7 \%$, os dados registrados em prontuário não coincidiram com o medicamento em uso referido pelo paciente, e 46 (63,9\%) pacientes referiram ter gastos mensais com medicamentos. Tais dados sugerem dificuldade de adesão ao tratamento prescrito e estímulo à automedicação, o que pode aumentar as taxas de mortalidade e morbidade, bem como a necessidade de internação hospitalar 18. Mion Jr. \& Pierin 19 encontraram que as principais causas para a baixa adesão ao tratamento anti-hipertensivo são relacionadas ao medicamento (custo, tomar várias vezes ao dia, efeitos colaterais), ao desconhecimento da gravidade da doença e a conhecimentos e crenças de saúde (uso do medicamento apenas quando aumenta a pressão arterial, falta de cuidado com a saúde, esquecimento, desconhecimento da gravidade da doença).

Neste estudo, 27 pacientes afirmaram receber todo medicamento em uso na USF em que fazem acompanhamento. Entre os 37 (57,8\%) indivíduos que referiram não receber todo medicamento em uso na USF, 31 (83,8\%) afirmaram comprar seu medicamento quando necessário, $4(10,8 \%)$ procuram o medicamento em outras USF ou até em municípios vizinhos, e 2 $(5,4 \%)$ esperam chegar o medicamento. Em relação ao recebimento do medicamento no local de atendimento, Halal et al. 20 encontraram uma probabilidade $33 \%$ maior de resolutividade nessa condição. Poder comprar o medicamento também esteve relacionado com maior resolutividade. Turrini 18 cita que a visita de rotina ao médico foi mais freqüente no grupo que recebeu a medicação gratuitamente.

Outra informação relevante, mesmo considerando o tamanho da amostra e o número de indivíduos insulino-dependentes (seis pessoas), é que três indivíduos afirmaram não receber todo o material necessário para a aplicação da insulina. Um dos pacientes referiu receber a insulina, uma seringa e uma agulha para cada três aplicações. Se a adesão ao tratamento está relacionada com a disponibilidade de medica-
Tabela 1

Acesso aos serviços de saúde antes da implantação do Programa Saúde da Família (PSF) no Município de Francisco Morato, São Paulo, Brasil, 2002.

\begin{tabular}{lrr}
\hline & N & $\%$ \\
\hline Acesso anterior a serviço de saúde & 47 & 73,4 \\
Sim & 17 & 26,6 \\
Não & 64 & 100,0 \\
Total & & \\
Qual serviço de saúde usava antes do PSF & & \\
Unidade básica de saúde/Hospitais em outros municípios & 25 & 53,2 \\
Unidade básica de saúde /Hospital no próprio município & 15 & 31,9 \\
Planos de saúde & 7 & 14,9 \\
Total & 47 & 100,0 \\
\hline
\end{tabular}

mentos, igualmente são necessárias condições para sua utilização e, no caso da insulinoterapia, há necessidade de seringa e agulha apropriadas. Apesar da recomendação de uso único na embalagem desses produtos, o Manual de Hipertensão Arterial e Diabetes Mellitus 10 afirma que, em boas condições de higiene, as mesmas podem ser utilizadas por até sete dias, sempre pela mesma pessoa. Souza 21 encontrou, em seu estudo, que $94,6 \%$ dos pacientes reutilizavam a seringa e a agulha. $\mathrm{O}$ autor cita que, com a reutilização das mesmas por quatro vezes, haveria redução de $74,6 \%$ nos custos com seringas e agulhas em insulinoterapia, ressaltando, porém, que são necessários estudos aprofundando a questão, "a fim de buscar na realidade brasileira evidências científicas que possam respaldar os profissionais de saúde em suas orientações aos portadores de diabetes" 21 (p. 48).

Dos indivíduos atendidos pelo PSF, $65,6 \%$ consideraram que houve resolutividade de seu problema pela ESF, e 68,8\% afirmaram estar satisfeitos com a atenção recebida na USF, porém, destes, $15,8 \%$ referiram como problema a falta de medicamentos. Entre os $31,2 \%$ de entrevistados insatisfeitos, os motivos são: falta de medicamentos, o mau atendimento, e em uma USF que estava passando por processo de mudança de local, a distância da nova unidade foi citada. A opinião dos entrevistados sobre a resolutividade do programa e sua satisfação com a assistência são apresentadas na Tabela 2.

Segundo Turrini 18 (p. 55), "a resolutividade dos serviços e a satisfação do cliente são maneiras de se avaliar os serviços de saúde, a partir dos resultados obtidos no atendimento ao usuário". Em se tratando de doenças crônicas, a ava- 
Tabela 2

Opinião dos entrevistados sobre a resolutividade do programa e satisfação com a assistência. Francisco Morato, São Paulo, Brasil, 2002.

\begin{tabular}{lrr}
\hline & N & $\%$ \\
\hline Houve resolutividade & & \\
Sim & 42 & 65,6 \\
Não & 22 & 34,4 \\
Total & 64 & 100,0 \\
& & \\
Considera-se satisfeito & & \\
Sim & 44 & 68,8 \\
Não & 20 & 31,2 \\
Total & 64 & 100,0 \\
\hline
\end{tabular}

liação do cliente dependerá do grau de aceitação e compreensão de sua doença. Em seu estudo, a referida autora encontrou $81,4 \%$ de satisfação com o atendimento do serviço e afirma que, quando o usuário se sente acolhido no serviço, provavelmente emitirá opinião de satisfação pelo atendimento.

\section{Qualidade da assistência}

Em relação à assistência, a Tabela 3 apresenta os indicadores propostos para análise da qualidade, com enfoque na consulta médica e de enfermagem. Cerca de $60 \%$ dos entrevistados tiveram sua primeira consulta com a ESF agendada em menos de 16 dias. O retorno foi agendado para $79,7 \%$ dos pacientes.

De acordo com as condições clínicas do paciente, a Sociedade Brasileira de Hipertensão ${ }^{6}$ recomenda o seguimento dos pacientes conforme o valor de pressão arterial encontrado, variando da intervenção imediata (hipertensão grave) a um ano (pressão arterial normal). Em relação ao diabetes mellitus, a Sociedade Brasileira de Diabetes 4 recomenda avaliação a cada três ou quatro meses para pacientes estáveis e com controle satisfatório.

A Sociedade Brasileira de Hipertensão 6 cita que os objetivos da investigação clínico-laboratorial do paciente hipertenso são a confirmação da elevação da pressão arterial, avaliação de lesões de órgãos-alvo, identificação de risco cardiovascular e diagnóstico da etiologia da hipertensão arterial, e que, para tal, são fundamentais a história clínica, o exame físico e a avaliação laboratorial. Da mesma forma, podemos atribuir à avaliação do paciente diabético os mesmos objetivos, relacionados à hiperglicemia e suas complicações.
Com essa fundamentação, analisou-se a qualidade da anamnese e do exame físico do conjunto de consultas feitas aos entrevistados, pelo médico ou pela enfermeira do PSF. Apenas sete pacientes passaram por exame físico considerado ótimo, e 23 passaram por anamnese ótima (Tabela 3 ).

Apesar de o PSF valorizar a assistência integral ao paciente por meio das consultas médicas e de enfermagem e do trabalho em grupos, com estímulo ao autocuidado, $34(53,1 \%)$ usuários do PSF referiram nunca ter participado de aulas, grupos ou palestras relacionados à sua patologia. Sobre as orientações que os entrevistados afirmaram ter recebido, a mais freqüente foi relacionada à dieta e, em seguida, relacionada à prática de atividade física, como aponta a Tabela 3. Spranger et al. 22 estudaram se as recomendações do VI Joint National Committee on Prevention, Detection, Evaluation, and Treatment od High Blood Presusre eram aplicadas na prática médica e perceberam que algumas condutas de diagnóstico e a terapêutica usada não condiziam com as recomendações.

Em estudo realizado por Guimarães \& Takayanagui 23 com pacientes inseridos no Programa de Assistência ao Diabético de uma unidade básica de Ribeirão Preto, São Paulo, verificou-se que, das prescrições médicas recebidas, apenas $17,2 \%$ abrangiam as recomendações da Sociedade Brasileira de Diabetes, incluindo informações sobre dieta, exercício físico e uso de medicamento.

Considerando apenas os indivíduos diabéticos do presente estudo, nenhum refere ter recebido orientações para realização do controle dos níveis de sua glicose através de fitas (glicemia capilar ou glicosúria), apesar de tal cuidado ser recomendado pela Sociedade Brasileira de Diabetes 3 e pelo Ministério da Saúde 10,24.

Dos 58 pacientes hipertensos entrevistados, $49(84,5 \%)$ referiram ter recebido orientação para verificação da pressão arterial fora dos períodos de consulta agendada. Destes, 40 $(81,6 \%)$ afirmaram procurar a USF quando necessário.

O tratamento do diabetes mellitus e da hipertensão arterial inclui orientação e educação em saúde, modificações no estilo de vida e, se necessário, o uso de medicamentos. As orientações são necessárias, tanto no que se refere ao tratamento medicamentoso quanto ao não medicamentoso. A educação em saúde é imprescindível, pois não é possível o controle adequado da glicemia e da pressão arterial se o paciente não for instruído sobre os princípios em que se fundamentam seu tratamento. A participação ativa do indivíduo é a única solução 
eficaz no controle das doenças e na prevenção de suas complicações 6,10,24.

\section{Estilo de vida}

Quanto à análise dietética, apenas 21 (32,8\%) dos 64 usuários do PSF entrevistados mostraram ter uma dieta adequada. Assunção et al. 8 também referem em um estudo baixa adesão à dieta, apesar da importância da dieta no controle do diabetes mellitus e da hipertensão arterial 3,6,10,25. Essa questão mostrou-se de difícil análise devido à possibilidade de o paciente poder não retratar fielmente seus hábitos alimentares. Ainda assim é importante na composição do perfil da população assistida.

A Tabela 4 mostra que 48 (75\%) indivíduos referiram não ter o hábito de praticar atividade física: $16(33,3 \%)$ por falta de tempo, $13(27,1 \%)$ por dores e cansaço, $6(12,5 \%)$ por causa das ruas serem íngremes, 3 (6,3\%) afirmaram não praticar atividade física devido ao diabetes mellitus ou à hipertensão arterial, e 10 (20,8\%) alegaram outros motivos. Siqueira 26 encontrou $41 \%$ de sedentarismo enquanto que Piccolomini 27 encontrou 62,7\%, Assunção et al. 8 também referem baixa adesão a exercícios físicos entre diabéticos no Sul do Brasil.

\section{Conclusão}

O PSF adotou, como prioridade na atenção ao adulto, ações de promoção, prevenção e tratamento de diabetes mellitus e hipertensão arterial, além de hanseníase e tuberculose. Este estudo apresenta, como seu principal resultado, o fato de que o Município de Francisco Morato conseguiu, com a implantação do PSF, ampliar o acesso da população aos serviços de atenção básica. Quase 27\% dos usuários do programa não usavam nenhum serviço de saúde antes da sua implantação, ainda que portadores de doenças crônicas. Entre os que já tinham acesso, cerca de $50 \%$ se deslocavam a outro município para tratamento.

O índice de satisfação dos usuários mostrou-se positivo, apesar das referidas queixas de falta de medicamento. Ao aferir se os procedimentos básicos de consulta, recomendados pelos consensos brasileiros de hipertensão arterial e diabetes mellitus, eram executados, percebeu-se que a questão da qualidade da assistência à saúde merece maior atenção. Apenas 7 $(10,9 \%)$ dos 64 usuários do PSF souberam reconhecer ou reconheceram que os procedimentos recomendados para um bom exame físico foram adotados em, pelo menos, uma consul-
Tabela 3

Dados referentes à consulta médica e de enfermagem segundo paciente atendido pelo Programa Saúde da Família (PSF). Francisco Morato, São Paulo, Brasil, 2002.

\begin{tabular}{|c|c|c|}
\hline & $\mathbf{N}$ & $\%$ \\
\hline \multicolumn{3}{|l|}{ Agendamento da 1a consulta (dias) } \\
\hline $0-7$ & 29 & 45,4 \\
\hline $8-15$ & 10 & 15,6 \\
\hline $16-30$ & 19 & 29,7 \\
\hline Acima de 30 & 6 & 9,3 \\
\hline Total & 64 & 100,0 \\
\hline \multicolumn{3}{|l|}{ Anamnese } \\
\hline Ótima & 23 & 36,0 \\
\hline Incompleta & 25 & 39,0 \\
\hline Insatisfatória & 16 & 25,0 \\
\hline Total & 64 & 100,0 \\
\hline \multicolumn{3}{|l|}{ Exame físico } \\
\hline Ótimo & 7 & 10,9 \\
\hline Incompleto & 29 & 45,3 \\
\hline Insatisfatório & 28 & 43,8 \\
\hline Total & 64 & 100,0 \\
\hline \multicolumn{3}{|l|}{ Orientações } \\
\hline Dieta hipocalórica & 38 & 59,4 \\
\hline Prática de atividade física & 35 & 54,7 \\
\hline Medidas de redução do estresse & 14 & 21,9 \\
\hline
\end{tabular}

Tabela 4

Dados referentes ao estilo de vida segundo paciente atendido pelo Programa Saúde da Família (PSF). Francisco Morato, São Paulo, Brasil, 2002.

\begin{tabular}{lrr}
\hline & N & $\%$ \\
\hline Dieta & & \\
Adequada & 21 & 33,3 \\
Parcialmente adequada & 27 & 42,2 \\
Inadequada & 15 & 23,8 \\
Total & 63 & 100,0 \\
Atividade física regular & & \\
Sim & & \\
Não & 16 & 25,0 \\
Total & 48 & 75,0 \\
\end{tabular}


ta, e 16 (25\%) deles tiveram sua anamnese incompleta. Estudo espanhol mostrou que a melhora da qualidade da assistência reduziu a proporção de pacientes com risco cardiovascular na comunidade estudada 28 . De fato, a melhoria da qualidade da assistência é uma preocupação na saúde pública mundial, posto que traz conseqüências importantes para o sucesso do tratamento e para a redução de danos.

Estudos de avaliação da assistência com enfoque na percepção da população são importantes e devem ser absorvidos pelos serviços como forma de melhorar o sistema. Os inquéritos populacionais mostram-se eficazes para qualificar a atenção básica, em especial, a de programas recém-implantados como o de Francisco Morato, apesar do custo relativamente alto e do cuidado metodológico necessário para seu desenvolvimento.

Alguns limites deste estudo merecem atenção. A topografia local do município estudado dificultou o acesso a algumas residências sorteadas, e o medo dos moradores, gerado pelo alto índice de violência local, fez com que alguns pacientes se recusassem a dar entrevistas. Apesar disso, a distribuição de sexo e faixa etária dos entrevistados mostrou-se semelhante à população cadastrada no SIAB.

\section{Resumo}

O Programa Saúde da Família (PSF) prioriza o atendimento a grupos considerados de maior risco a agravos, entre eles, a população com diabetes e hipertensão. Este estudo tem como objeto avaliar a assistência à população usuária do PSF de Francisco Morato, São Paulo, Brasil. Desenvolveu-se um inquérito domiciliar, com amostra probabilística dos pacientes com diabetes elou hipertensão cadastrados no município. A análise enfocou questões referentes ao acesso, à satisfação do usuário e à qualidade da assistência. Dos 72 entrevistados, 14 eram diabéticos, 30 hipertensos e 28 portadores das duas patologias. Do total de entrevistados, 64 (88,9\%) eram usuários do PSF local. O estudo destaca que 26,6\% desses usuários não tinham acesso a nenhum serviço de saúde antes da implantação do PSF. Entre os que tinham acesso, 53,2\% se deslocavam a outro município. Os índices de satisfação do usuário e percepção da resolutividade do programa mostraramse favoráveis, em torno de 66\%. Por outro lado, 57,8\% dos entrevistados relataram que não recebem todo o medicamento do serviço. Quanto à qualidade da assistência, detectou-se que $25 \%$ das anamneses eram incompletas, e 43,7\% dos exames físicos eram insatisfatórios. O PSF mostrou-se uma alternativa válida para aumentar o acesso à saúde, na busca de maior eqüidade no atendimento às necessidades da população.

Hipertensão; Diabetes Mellitus; Assistência ao Paciente

\section{Colaboradores}

Todos os autores participaram no delineamento do artigo, análise dos dados e redação final. 


\section{Referências}

1. Ministério da Saúde. Doenças cardiovasculares no Brasil. Sistema Único de Saúde - SUS: dados epidemiológicos, assistência médica. Brasília: Coordenação de Doenças Cardiovasculares, Ministério da Saúde; 1993.

2. Ministério da Saúde. Doenças cardiovasculares. http://www.saude.gov.br\programas \cardio.htm. (acessado em 08/Ago/2001).

3. Sociedade Brasileira de Diabetes. Consenso brasileiro sobre diabetes: diagnóstico e classificação de diabetes mellitus e tratamento do diabetes mellitus tipo 2. São Paulo: Sociedade Brasileira de Diabetes; 2000.

4. Sociedade Brasileira de Diabetes. Detecção e tratamento das complicações crônicas do diabetes mellitus, 1998. http://www.diabetes.org.br (acessado em 15/Mai/2001).

5. Departamento de Atenção Básica, Secretaria de Políticas de Saúde. Programa Saúde da Família. Rev Saúde Pública 2000; 34:316-9.

6. Sociedade Brasileira de Hipertensão. III Consenso Brasileiro de Hipertensão Arterial. São Paulo: Sociedade Brasileira de Hipertensão; 1998.

7. Barnett AH, Dodson M. Hypertension and diabetes. London: Science Press; 1997.

8. Assunção MCF, Santos IS, Gigante PD. Atenção primária em diabetes no Sul do Brasil: estrutura, processo e resultado. Rev Saúde Pública 2001; 35:88-95.

9. Ministério da Saúde. A implantação da Unidade de Saúde da Família. Brasília: Secretaria de Políticas de Saúde, Ministério da Saúde; 2000.

10. Ministério da Saúde. Manual de hipertensão arterial e diabetes mellitus. Brasília: Secretaria de Políticas de Saúde, Ministério da Saúde; 2002.

11. Contandriopoulos AP, Champagne F, Denis JL, Pineault R. A avaliação na área da saúde: conceitos e métodos. In: Hartz ZMA, organizadora. Avaliação em saúde: dos modelos conceituais à prática na análise da implantação de programas. Rio de Janeiro: Editora Fiocruz; 1997. p. 29-48.

12. Cesar CLC, Tanaka O. Inquérito domiciliar como instrumento de avaliação de serviços de saúde. Cad Saúde Pública 1996; 12 Suppl 2:59-70.

13. Lwanga SK, Lemeshow S. Sample size determination in health studies. Geneva: World Health Organization; 1991.

14. Silva NN. Amostragem probabilística. São Paulo: Edusp; 1998.

15. Fórum Metropolitano de Segurança Pública. Estatísticas de Francisco Morato. http://www.forumsorg.br/perfil_francisco_morato.htm (acessado em 01/Mar/2003).

16. Diamant AL, Brook RH, Fink A, Gelberg L. Assessing use of primary health care services by very low-income adults in a managed care program. Arch Intern Med 2001; 161:1222-7.
17. Araújo RB, Santos I, Cavaleti MA, Costa JSD, Béria JU. Avaliação do cuidado prestado a pacientes diabéticos em nível primário. Rev Saúde Pública 1999; 33:24-32.

18. Turrini RNT. Percepção dos usuários sobre a resolutividade e a satisfação pelos serviços de saúde na região sudoeste da Grande São Paulo [Tese de Doutorado]. São Paulo: Escola de Enfermagem, Universidade de São Paulo; 2001.

19. Mion Jr. D, Pierin AMG. Causas de baixa adesão ao tratamento e perfil de pacientes hipertensos. Coração e Circulação 1996; 7:69-72.

20. Halal IS, Sparrenberger F, Bertoni AM, Ciacomet C, Seibel CE, Lahude FM, et al. Avaliação da qualidade de assistência primária à saúde em localidades urbanas na região Sul do Brasil. Rev Saúde Pública 1994; 28:131-6.

21. Souza CR A prática da utilização de seringas descartáveis na administração de insulina no domicílio [Dissertação de Mestrado]. Ribeirão Preto: Escola de Enfermagem, Universidade de São Paulo; 1999.

22. Spranger CB, Ries AJ, Berge CA, Radford NB, Victor RG. Identifying gaps between guidelines and clinical practice in the evaluation and treatment of patients with hypertension Am J Med 2004; 117:14-8.

23. Guimarães FPM, Takayanagui AMM. Orientações recebidas do serviço de saúde por pacientes para o tratamento do portador de diabetes mellitus tipo 2. Rev Nutr 2002; 15:37-44.

24. Secretaria de Assistência à Saúde. Manual de diabetes. Brasília: Secretaria de Assistência à Saúde, Ministério da Saúde; 1993.

25. National Institutes of Health. Joint National Committee: on prevention, and treatment of high blood pressure. Bethesda: National Institutes of Health; 1997. (Publicação NIH, 98 - 4080).

26. Siqueira FPC. Estilo de vida e hipertensão [Dissertação de Mestrado]. Ribeirão Preto: Escola de Enfermagem de Ribeirão Preto, Universidade de São Paulo; 2002.

27. Piccolomini AF. Caracterização de indivíduos com diabetes mellitus matriculados em duas unidades básicas de saúde de Piracicaba [Dissertação de Mestrado]. São Paulo: Faculdade de Saúde Pública, Universidade de São Paulo; 2002.

28. Garcia-Ortiz L, Santos-Rodriguez I, Sanchez-Fernandez F, Mora-Santiago MC, Arganda-Maya J, Rodríguez-Corral MT. Efectividad de una intervención de mejora de calidad en la reducción del riesgo cardiovascular en pacientes hipertensos. Rev Esp Cardiol 2004; 57:644-51.

Recebido em 15/Dez/2003

Versão final reapresentada em 18/Mai/2005

Aprovado em 20/Jul/2005 